\title{
Vulnerabilidad por erosión fluvial: caso del corregimiento de Palo de Agua, Municipio de Lorica, Departamento de Córdoba, Colombia
}

\author{
Vulnerability due to fluvial erosion: case of the Palo de Agua \\ township, Municipality of Lorica, Department of Córdoba, Colombia
}

Orlando Tordecilla-Correa ${ }^{1}$ Environment \& Technology Foundation, Colombia

Rubén Darío Sepulveda-Vargas ${ }^{3}$ Universidad Pontificia Bolivariana, Montería, Colombia Ana Michelle Gómez-Constante 5 Environment \& Technology Foundation, Colombia,
Jorge Villadiego-Lorduy ${ }^{2}$ Universidad Pontificia Bolivariana,

Montería, Colombia Lilliana Piedra-Castro ${ }^{4}$ Environment \& Technology Foundation, Colombia, Universidad Nacional, Costa Rica Javier Sánchez-Castillo ${ }^{6}$ Universidad Pontificia Bolivariana Montería, Colombia

1 Geógrafo. Magister en Ciencias Ambientales. Asesor científico Environment \& Technology Foundation. Correo electrónico: etf@environmenttechnologyfoundation.org.

(i) https://orcid.org/0000-0002-3449-3953.

2 Doctor en Ciencias Naturales y Desarrollo Sustentable. Docente Investigador Universidad Pontificia Bolivariana, sede Montería, Colombia. Coordinador del Grupo de Investigación CAMHA. Correo electrónico: Jorge.villadiegol@upb.edu.co. (D) https://orcid.org/0000-0002-6151-9987.

3 Doctor en Ciencias Sociales. Docente Investigador Universidad Pontificia Bolivariana, sede Montería. Colombia. Correo electrónico: ruben.sepulveda@upb.edu.co. (D) https://orcid.org/0000-0001-6694-3907.

4 Doctora en Ciencias Naturales para el Desarrollo. Laboratorio de Recursos Naturales y Vida Silvestre (LARNAVISI). Environment \& Technology Foundation-Colombia, Universidad Nacional, Costa Rica. Correo electrónico:1illiana.piedra.castro@una.ac.cr etf@environmenttechnologyfoundation.org. https://orcid.org/0000-0003-4878-1531

5 Ingeniera Sanitaria y Ambiental. Asesor ambiental. Environment \& Technology Foundation. Colombia. Correo electrónico: etf@environmenttechnologyfoundation.org.

(iD https://orcid.org/0000-0003-3847-7114

6 M.Sc. Ing. Metalúrgico y de Materiales. Docente Investigador Universidad Pontificia Bolivariana, sede Montería, Colombia. Coordinador del Grupo de Investigación OPUREB.

Correo electrónico: javier.sanchezc@upb.edu.co. (D) https://orcid.org/0000-0001-8570-7053 


\begin{abstract}
Resumen
El presente estudio evalúa la vulnerabilidad del centro poblado en el corregimiento de Palo de Agua, asentado a orillas del río Sinú perteneciente al municipio de Santa Cruz de Lorica, Colombia. Se aplica una metodología de enfoque mixto con técnicas de entrevista a actores claves y cálculos cuantitativos para evaluar la vulnerabilidad total, física, ambiental, económica y social, a partir del reconocimiento en campo y del análisis de procesos por erosión fluvial en puntos críticos; sin omitir las amenazas y la estimación del riesgo para el corregimiento en mención. Se infiere que la vulnerabilidad total media es la categoría de mayor predominancia con un $84 \%$.
\end{abstract}

Palabras clave: vulnerabilidad, erosión fluvial, inundaciones, amenazas, riesgo.

\begin{abstract}
The present study assesses the vulnerability of the populated center in the township of Palo de Agua, located on the banks of the Sinú River, belonging to the municipality of Santa Cruz de Lorica, Department of Córdoba, Colombia. A mixed approach methodology is followed, with interview techniques applied to key actors and also by means of quantitative calculations to evaluate total, physical, environmental, economic and social vulnerabilities, based on field recognition data and fluvial erosion processes data analysis gathered from critical geographical points; without omitting to consider hazards and risk estimation applicable to Pablo de Agua township. It is inferred that the average total vulnerability constitutes the predominant vulnerability category at $84 \%$ level.
\end{abstract}

Keywords: Vulnerability; Fluvial erosion; Floods; Hazards; Risk.

\title{
Introducción
}

Los eventos reiterativos de amenazas por inundaciones y la formación de puntos críticos por procesos de erosión fluvial en el bajo Sinú, Colombia, en el corregimiento de Palo de Agua, municipio de Lorica, son motivos de preocupación por la vulnerabilidad social y ambiental que afecta de forma directa la calidad de vida de los moradores.

La vulnerabilidad de la población por el fenómeno de la erosión fluvial constituye un procedimiento complejo que incluye la identificación, caracterización y análisis de las condiciones de amenaza con el fin de precisar las propiedades y valores que permitan estimar el nivel de daño del evento (Euroclima, 2014, p.51).

Existe una relación intrínseca entre la marginalidad económica y la vulnerabilidad, por lo que el estudio de factores socioeconómicos de familias, son las dimensiones más relevantes a la hora de evaluar y determinar la vulnerabilidad (Programa de Naciones Unidas para el Desarrollo (PNUD), 2014, p.2). La vulnerabilidad global es un concepto propuesto por Wilches (1993) y estudiado en países como Cuba desde el año 2008 en 
cabeza de la Agencia de Medio Ambiente (AMA), resaltándose dentro de estas investigaciones a Yaima (2018), quien asocia la evolución cualitativa de las condiciones naturales y sociales de las poblaciones, respecto a la magnitud de los fenómenos de erosión e inundaciones.

Según la Oficina de las Naciones Unidas para la Reducción del Riego de Desastres (UNISDR, 2015, p.62) indica que Colombia en el contexto Latinoamérica registra la tasa más alta de desastres recurrentes provocados por fenómenos naturales, con más de 600 eventos reportados cada año en promedio y el décimo lugar de riesgo económico por desastres naturales en el mundo, donde el $84,7 \%$ de la población y el 86,6 \% de los activos, están localizados en áreas expuestas a dos o más peligros naturales.

El Departamento Nacional de Planeación (2012. p.25) describe el riesgo como la probabilidad de que ocurran pérdidas socioeconómicas y ecosistémicas producto de la materialización de eventos climatológicos extremos, atmosféricos, geológicos, volcánicos, tecnológicos, químicos, ambientales, entre otros, para lo cual se debe tener en cuenta los impactos negativos que se han generado en la comunidad durante los últimos años, evaluando las lluvias, sequías y vientos.

Por su parte, la amenaza o peligro se considera un proceso, fenómeno o actividad humana que puede ocasionar muertes, lesiones u otros afectos en la salud, daños a los bienes, disrupciones sociales y económicas o daños ambientales (Estrategia Internacional para la reducción de desastres de las Naciones Unidas (UNISDR, 2009). Es decir, se convierte en una amenaza determinado evento cuando hay factores expuestos que puedan verse afectados sea un bien y/o comunidad. Estos se pueden dividir según su origen como amenazas antrópicas y amenazas naturales.

Con base en lo anterior, se indica que la vulnerabilidad es la predisposición de las comunidades, bienes sociales, económicos o ambientales que puedan sufrir daño por la ocurrencia del fenómeno amenazante debido a sus condiciones de magnitud y duración.

Es importante resaltar que existen diversos tipos de vulnerabilidad que depende de factores físicos, ambientales, económicos y sociales, que permiten cuantificar el grado de susceptibilidad del sistema. Esta varía considerablemente dentro de una comunidad y con el transcurso del tiempo.

Por lo tanto, se identifica la vulnerabilidad como una característica de los elementos de interés (comunidad, sistema o bien) lo cual es 
independiente de su exposición (UNISDR, 2009, p.17). Así el riesgo se constituye como una relación dinámica entre la amenaza y la vulnerabilidad, el cual ha llegado a conceptualizarse para la evaluación cuantitativa del estudio.

En este orden de ideas, la investigación buscó evaluar la vulnerabilidad del centro poblado del corregimiento de Palo de Agua, asentado a orillas del río Sinú pertenecientes al municipio de Santa Cruz de Lorica, en Colombia, con el fin de aportar al debate científico local y contribuir a la formulación de políticas públicas de gestión riesgo.

\section{Metodología}

La población universo de esta investigación estuvo determinada por 37 manzanas en el corregimiento de Palo de Agua. Los criterios utilizados para la toma de la muestra se centraron en la distribución predial y el total del área urbana del centro poblado. Se utilizó un muestreo estadístico aleatorio simple con un tamaño muestral de 24 manzanas, para la aplicación de un cuestionario de 24 preguntas, tomando una vivienda por manzana. Cabe resaltar, que el instrumento en mención se soportó en la Guía Metodológica para la Elaboración de Planes Departamentales de Gestión del Riesgo, elaborada por el Programa de Naciones Unidas para el Desarrollo Colombia y la Unidad Nacional de Gestión de Riesgo y de Desastres (PNUD \& UNGRD, 2012, p.13) y diseñado para obtener información a nivel de organización, participación, grado de relación y conocimiento del riesgo, edad de la vivienda, materiales de construcción, entre otras variables.

Además, se realizaron entrevista semiestructurada referentes a la gestión del riesgo a funcionarios del sector público de la Alcaldía de Lorica y de la Corporación Autónoma Regional de los Valles del Sinú y San Jorge (CVS), como también a miembros integrantes de la Asociación de Productores para el Desarrollo Comunitario del bajo Sinú (ASPROCIG) y de la Corporación ecoturística y biodiversidad de Bañó (ECONBIBA).

Adicional a esto, en campo se verificaron los puntos críticos aledaños al río y las condiciones sanitarias de la vivienda y su tipología. Se desarrolló un análisis estadístico de tipo descriptivo para cada una de las variables analizadas en el cuestionario utilizando el software R (2015) y Excel (2010). 
Para el análisis de vulnerabilidad se trabajó con cartografía del Marco Geoestadístico Nacional (MGN) del Departamento Nacional de Estadística (DANE, 2017) a escala 1:25.000 y cartografía base oficial del Instituto Geográfico Agustín Codazzi (IGAC, 2016) a escala 1:25.000.

Se utilizaron imágenes satelitales Airbus con resolución espacial de un metro, año 2016. Dentro de los softwares aplicados para el procesamiento geográfico y toma de datos en campo se usó ArcGis 10.3 y GPS Garmin 60sc.

En lo referente a la estimación de la vulnerabilidad se analizó y calificó cada variable asignándole un valor numérico de 1 a 3 basado en los criterios de la Guía Metodológica para la elaboración de Planes Departamentales para la Gestión del Riesgo (PNUD \& UNGRD, 2012, p.29), con el fin de agruparlas en nivel bajo, medio y alto respectivamente, para luego sumar cada uno de los valores dando como resultado un estimado ponderado para cada dimensión de vulnerabilidad evaluada.

El cálculo de la vulnerabilidad total se realizó empleando la ecuación uno propuesta por el Programa de Naciones Unidas para el Desarrollo Colombia (PNUD) y la Unidad Nacional de Gestión de Riesgo y de Desastres (UNGRD, 2012, p. 36).

$$
V t=V f+V a+V e+V s \quad \text { Ecuación } 1
$$

dónde: $\boldsymbol{V t}$ : vulnerabilidad Total, Vf: Vulnerabilidad Física, Va: Vulnerabilidad Ambiental, Ve: Vulnerabilidad Económica y Vs: Vulnerabilidad Social.

Los niveles de riesgo se definieron de forma cualitativa empleando una matriz de doble entrada que permitió relacionar los grados de amenaza y vulnerabilidad empleando el método de superposición de capas, para determinar los niveles de riesgo presentes.

De este modo el color verde representa el riesgo bajo (intervalos de 16 a 26), el amarillo el medio (intervalos de 27 a 37) y el rojo el alto (intervalos de 38 a 48), como se muestra en la sesión de resultados.

Además de la información capturada con el cuestionario, se acudió a documentos de información secundaria del orden internacional para la gestión de riesgo de desastres (Cáritas del Perú, 2009, p.17), incidencia de la erosión en la vulnerabilidad (Ochoa, 2011, p.45). 
En el contexto local los estudios sobre diagnóstico y evaluación de amenazas y riesgo (CVS \& Universidad del Sinú, 2008; CVS \& Universidad Escuela de Administración, Finanzas e Instituto Tecnológico (EAFIT, 2012).

\section{Resultados}

El área de estudio de esta investigación se realizó en el corregimiento de Palo de Agua, municipio de Lorica, Colombia, localizado en las coordenadas geográficas latitud 9 9'33,59'N longitud 7550'40,13”O. El corregimiento está representado por un núcleo poblado, con un especial desarrollo por su ubicación geográfica y condiciones de accesibilidad.

Figura 1. Localización espacial corregimiento de Palo de Agua

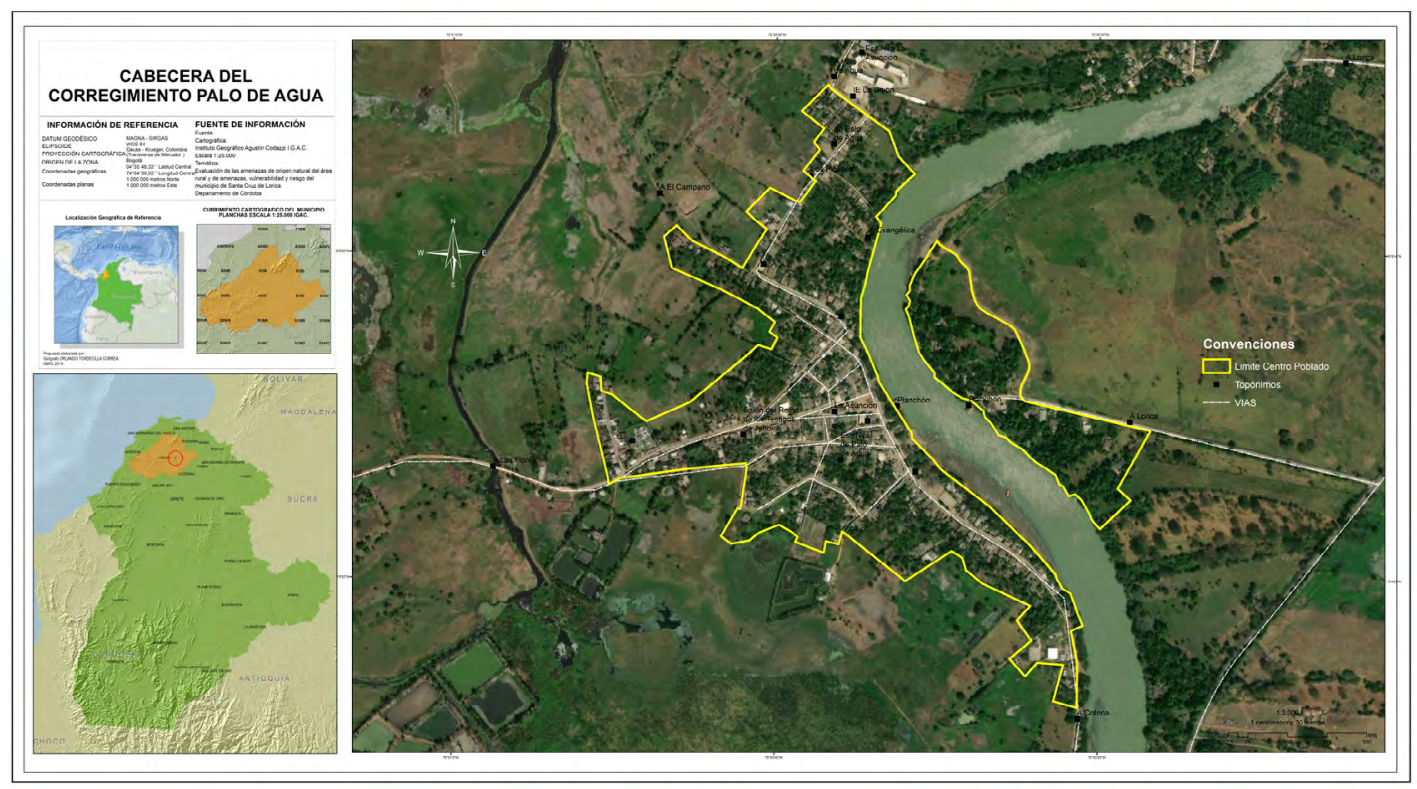

Fuente: Google Earth (2016).

Las características económicas del área de estudio describen hogares con ingreso monetario menor a un salario mínimo mensual (\$980.657) para el sostenimiento de familias con un promedio de cuatro integrantes.

Este aspecto además de la estacionalidad laboral como jornalero en actividades agrícolas transitorias y en la extracción de material de arrastre en la ribera del río, simbolizan los bajos condiciones de calidad de vida de 
los moradores, sin omitir el escaso acceso al mercado laboral, en muchos casos debido al bajo nivel educativo.

En lo que respecta a los servicios públicos, la población objeto de esta investigación tienen accesibilidad del $100 \%$ de servicios de acueducto y energía eléctrica, sin embargo, para el caso del gas natural, recurren a métodos tradicionales como fogón de leña, por no contar con cobertura del servicio.

El 54\% de las edificaciones en Palo de Agua tienen una antigüedad de 20 años de construida cuyas características estructurales son principalmente en bloque, grietas en los cerramientos de los pisos, techos en teja de fibrocemento, desgaste en las paredes y estructuras frágiles construidas con materiales de fácil deterioro como tierra y guadua.

Por otra parte, el $20 \%$ de las viviendas aún conservan estructuras en bahareque y/o guadua con techos de palma. El 17\% de las edificaciones del corregimiento en mención presenta estructuras con materiales y técnicas constructivas precarias.

Un 33\% de las viviendas del área de estudio, están sobre la zona de protección del drenaje y sus alrededores (entre 0 y 50 metros el cauce), caracterizándose por una alta vulnerabilidad a condiciones de riesgo.

Estas edificaciones no cumplen con los aspectos mínimos considerados por el Ministerio de ambiente, Vivienda y Desarrollo territorial (2010) en el Reglamento Colombiano de Construcción Sismo Resistente (NSR-10), es decir, el 50\% de las viviendas de Palo de Agua no cumplen con la Norma Técnica, relacionando esta condición con un posible riesgo sísmico.

En este sentido, es importante resaltar las condiciones de estabilidad en el corregimiento, debido a que son suelos colapsables, clase II, encontrando entre sus órdenes, entisoles, inceptisoles, alfisoles y molisoles, según el Sistema Taxonómico Norteamericano; suelos que presentan pendientes menores al 7\% y un drenaje que puede clasificar de pobre a muy pobre, con un nivel freático alto y porosidad abundante, propiciando altas concentraciones de humedad y poca estabilidad.

La geología del sector se encuentra conformada por depósitos aluviales (Qal), asociados a la dinámica de río Sinú, sus características geomorfológicas se encuentra dada por geoformas de tipo aluvial, esencialmente llanuras de inundación específicamente en la zona donde se localiza el centro poblado. 
En campo se evidenció un moderado contenido de materia orgánica en descomposición y amenazas por procesos de erosión fluvial e inundaciones, las inundaciones en el municipio de Lorica se encuentran influenciadas por cambios en las dinámicas hidrológicas del río Sinú y las fuertes precipitaciones que se han registrado desde la década de los años 2000 (Defensoría del Pueblo, 2005, p.34 ; Sepúlveda, 2015, p.11).

Históricamente algunos de los corregimientos más afectados por las inundaciones en el municipio de Lorica ha sido precisamente Palo de Agua dado a su proximidad espacial con el rio Sinú y cuerpos de agua receptores, además de la presencia de áreas críticas afectadas por erosión fluvial.

Las unidades de análisis que se usaron para este tipo de amenazas fueron la geomorfología, específicamente en el caso de las unidades de origen aluvial y fluvial - lacustre. La amenaza por inundación que persiste en esta región es alta, indicando que permanecen inundadas y/o presentan temporalidad de inundación media alta y alta, caracterizadas por ser áreas de inundación natural (CVS- EAFIT, 2012) (Figura 2).

Figura 2. Amenaza por inundación corregimiento de Palo de Agua

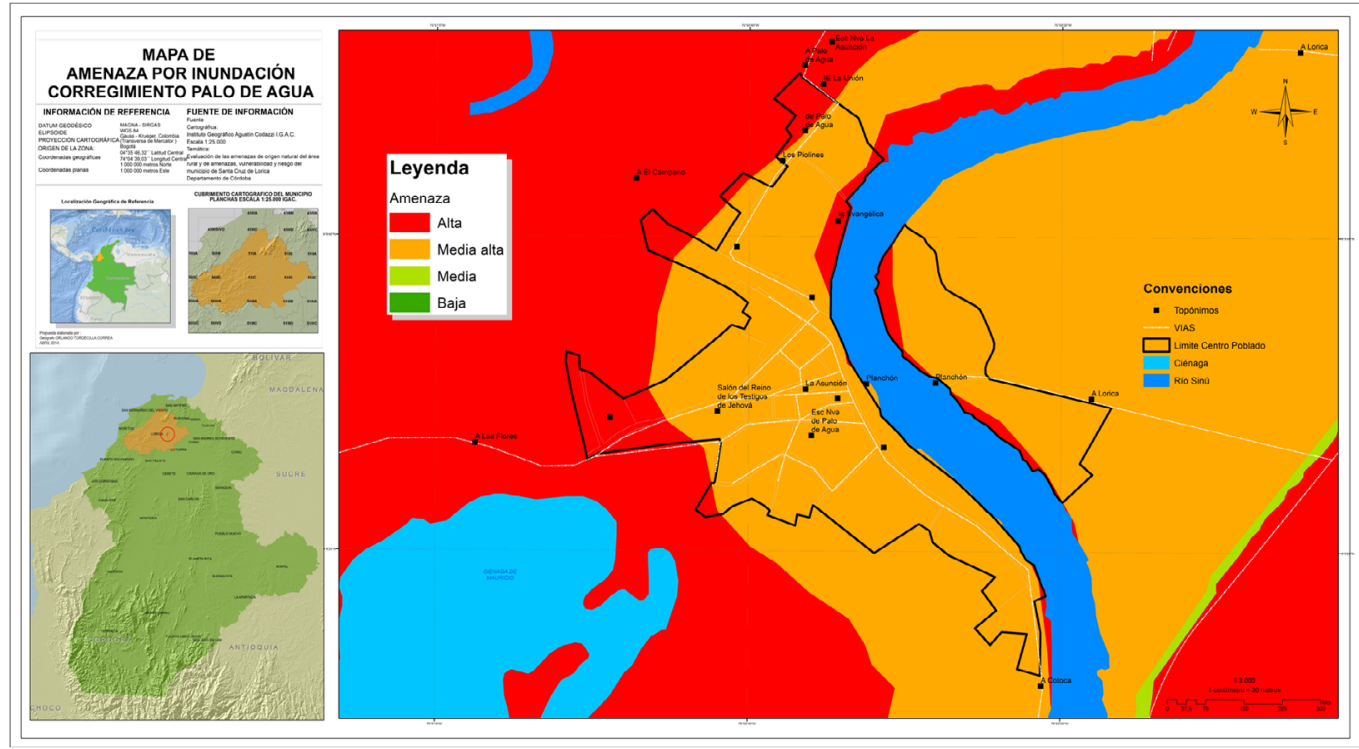

Fuente: Temática CVS- EAFIT, 2012. Diseño cartográfico elaboración propia (2016). 


\section{Procesos de erosión fluvial y puntos críticos}

En el municipio de Lorica se encuentran localizados 37 puntos críticos de los cuales uno tiene influencia en el corregimiento de Palo de Agua, ubicados sobre la margen izquierda del río Sinú, a la altura de Boca Nicolasa (Tabla 1).

Tabla 1: Ubicación punto crítico corregimiento Palo de Agua

\begin{tabular}{|c|c|c|c|c|}
\hline Margen & Centro poblado & Nombre punto critico & X & Y \\
\hline \multirow{2}{*}{ Izquierda } & Palo de Agua & Boca Nicolasa - inicia & 805940,0723 & 1505295,231 \\
\cline { 2 - 5 } & Palo de Agua & Boca Nicolasa - finaliza & 806096,3505 & 1505522,43 \\
\hline
\end{tabular}

Fuente: Elaboración propia (2016).

Las situaciones evidenciadas en los puntos críticos para el año 2014, presentan una variación con las identificadas en campo en el periodo de estudio 2016 y actualizadas a 2019.

Igualmente, el trabajo en terreno permitió poner en evidencia la formación de un nuevo punto crítico en el corregimiento de Palo de Agua. Según los pobladores del sector, se ha formado por el tránsito de vehículos pesados que circulaban por la vía, esto indica que la formación del punto crítico tiene un origen antrópico (Figura 3).

Figura 3. Punto crítico en formación corregimiento de Palo de Agua.

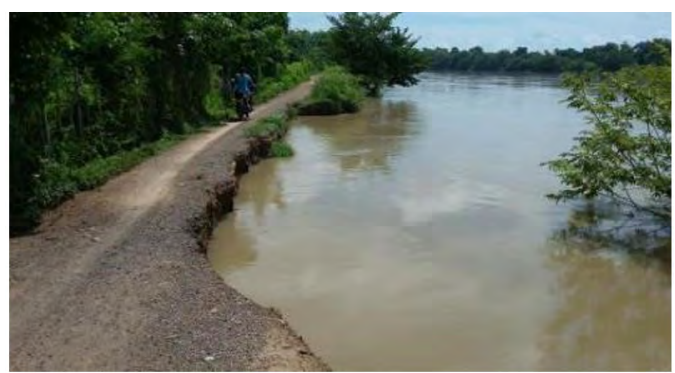

Fuente: Elaboración propia (2016).

\section{Estimación de la vulnerabilidad total y por dimensiones}

Para la estimación de la vulnerabilidad total y por dimensiones se consideró los criterios establecidos en la guía metodológica para la elaboración de planes departamentales para la gestión del riesgo citado arriba. 
El nivel de vulnerabilidad del corregimiento de Palo de Agua resultó de la incidencia de los factores sociales, físicos, económicos y ambientales. Para el cálculo de vulnerabilidades, incluyendo la total, se obtuvieron valores producto de la aplicación del cuestionario de caracterización a 24 viviendas en el corregimiento de Palo de Agua, que evaluaban las dimensiones físicas, ambientales, económicas y sociales y las correspondientes subvariables para cada una.

Para la dimensión física, los valores limites inferior y superior derivados de la evaluación oscilaban entre 8 y 15, referente a la dimensión económica éstos se encontraban entre 7 y 11 .

De igual forma, la dimensión ambiental presentó valores entre 3 y 4 , por último, la dimensión social registró valores entre 4 y 8 . Como resultado de las sumatorias en vertical de cada una de las dimensiones se obtuvo la vulnerabilidad total para cada vivienda frente a la amenaza por erosión fluvial.

En este caso en particular, los valores de la vulnerabilidad total calculada fluctuaban entre 25 a 34 clasificándose únicamente en dos categorías: vulnerabilidad media y baja (Tabla 2).

La vulnerabilidad total baja es la de menor incidencia en el centro poblado de Palo de Agua y comprende el 16\% del área urbana del corregimiento, la población que reside en esta zona presenta mejores condiciones en las variables evaluadas que el resto de los habitantes del corregimiento, denotando mejorías en las infraestructuras de las viviendas, ingresos económicos y empleos fijos, así como una participación con la comunidad y las organizaciones presentes.

La vulnerabilidad total media, es la segunda categoría que se identificó en el centro poblado Palo de Agua, siendo la de mayor extensión en ocupación con el $84 \%$ del área urbana. La vulnerabilidad económica y la baja capacidad de ingresos que presenta la población del corregimiento el cual se encuentra asociado a las pocas oportunidades laborales, además de la baja capacidad de la infraestructura a soportar ciertas condiciones de amenaza, son los factores que determinan esta zona. (Figura 4). 
Orlando Tordecilla-Correa, Jorge Villadiego-Lorduy, Rubén Dario Sepulveda-Vargas,

Lilliana Piedra-Castro, Ana Michelle Gómez-Constante

Vulnerability due to fluvial erosion: case of the Palo de Agua township, Municipality of Lorica,

Department of Córdoba, Colombia.

Tabla 2: Matriz de evaluación de vulnerabilidad total y por dimensiones del corregimiento Palo de Agua.

\section{MATRIZ DE EVALUACIÓN DE VULNERABILIDADES}

\begin{tabular}{|c|c|c|c|c|c|c|c|c|c|c|c|c|c|c|c|c|c|c|c|c|c|c|c|c|}
\hline DIMENSIÓN FÍSICA & E1 & E2 & E3 & E4 & E5 & E6 & E7 & E8 & Eg & E10 & E11 & E12 & E13 & E14 & E15 & E16 & \begin{tabular}{|l|l|} 
E17 \\
\end{tabular} & E18 & E19 & E20 & E21 & E22 & E23 & E24 \\
\hline Antigüedad de la edificacion & 2 & 2 & 2 & 3 & 3 & 3 & 3 & 3 & 3 & 3 & 2 & 2 & 3 & 3 & 2 & 2 & 3 & 3 & 3 & 3 & 3 & 2 & 2 & 3 \\
\hline Materiales de construccion & 1 & 1 & 2 & 3 & 3 & 3 & 3 & 3 & 2 & 2 & 2 & 1 & 1 & 1 & 1 & 2 & 2 & 3 & 3 & 3 & 2 & 2 & 2 & 1 \\
\hline Cumplimiento de la normatividad vigente & 2 & 2 & 3 & 3 & 3 & 3 & 3 & 3 & 3 & 2 & 2 & 1 & 1 & 1 & 1 & 2 & 2 & 3 & 3 & 3 & 2 & 3 & 3 & 2 \\
\hline Caracteristicas geologicas y tipo de suelo & 1 & 3 & 3 & 3 & 3 & 3 & 3 & 3 & 3 & 3 & 3 & 3 & 3 & 3 & 3 & 3 & 1 & 2 & 2 & 1 & 3 & 3 & 3 & 3 \\
\hline $\begin{array}{l}\text { Localizacion de las edificaciones con } \\
\text { respecto a las zonas de retiro a fuentes de } \\
\text { agua y zonas de riesgo }\end{array}$ & 2 & 3 & 3 & 3 & 3 & 3 & 3 & 3 & 2 & 2 & 2 & 2 & 2 & 2 & 2 & 3 & 1 & 1 & 1 & 1 & 1 & 1 & 1 & 2 \\
\hline TOTAL & 8 & 11 & 13 & 15 & 15 & 15 & 15 & 15 & 13 & 12 & 11 & 9 & 10 & 10 & 9 & 12 & 9 & 12 & 12 & 11 & 11 & 11 & 11 & 11 \\
\hline DIM & 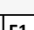 & & $\sigma_{0}$ & & 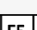 & & & & & & & $r^{2}$ & & Fia & & or & & 510 & & & & & & \\
\hline \begin{tabular}{|l} 
Situacion de pobreza y seguridad \\
\end{tabular} & E1 & E2 & E3 & \begin{tabular}{|l|l} 
E4 & \\
\end{tabular} & Es & \begin{tabular}{|c|c}
6 \\
\end{tabular} & 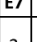 & \begin{tabular}{|l|l} 
E8 \\
\end{tabular} & $E 9$ & E10 & & EL12 & & 514 & & 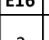 & & & & & & 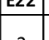 & & \\
\hline alim & 2 & 2 & 3 & 3 & 2 & 2 & 3 & 3 & 3 & 3 & 3 & 2 & 1 & 2 & 1 & 2 & 3 & 1 & 3 & 2 & 2 & 2 & 3 & 2 \\
\hline Nivel de ingresos & 3 & 3 & 3 & 3 & 3 & 3 & 3 & 3 & 3 & 2 & 3 & 2 & 1 & 2 & 1 & 2 & 3 & 2 & 3 & 3 & 2 & 2 & 3 & 2 \\
\hline ervicios publicos & 2 & 2 & 2 & 2 & 2 & 2 & 2 & 2 & 2 & 3 & 2 & 2 & 2 & 2 & 2 & 2 & 2 & 2 & 2 & 2 & 2 & 2 & 2 & 2 \\
\hline accesoal & 3 & 3 & 3 & 3 & 3 & \begin{tabular}{|l|l|}
3 \\
\end{tabular} & 3 & 3 & 3 & 3 & 3 & 3 & 3 & 3 & 3 & 3 & 3 & 3 & 3 & 3 & 3 & 3 & 3 & 3 \\
\hline TOTAL & 10 & 10 & 11 & 11 & 10 & 10 & 11 & 11 & 11 & 11 & 11 & 9 & 7 & 9 & 7 & 9 & 11 & 8 & 11 & 10 & 9 & 9 & 11 & 9 \\
\hline \begin{tabular}{|l} 
DIMENSIÓN AMBIENTAL \\
\end{tabular} & E1 & E2 & E3 & E4 & E5 & \begin{tabular}{|l|l|} 
E6 \\
\end{tabular} & E7 & E8 & E9 & \begin{tabular}{|l|l|} 
E10 \\
\end{tabular} & \begin{tabular}{|l|l|} 
E11 \\
\end{tabular} & \begin{tabular}{|l|l|} 
E12 \\
\end{tabular} & E13 & \begin{tabular}{|l|l} 
E14 \\
\end{tabular} & E15 & \begin{tabular}{|l|l} 
E16 \\
\end{tabular} & E17 & E18 & \begin{tabular}{|l|l|} 
E19 \\
\end{tabular} & \begin{tabular}{|l|l|} 
E20 \\
\end{tabular} & E21 & \begin{tabular}{|l|l|} 
E22 \\
\end{tabular} & E23 & E24 \\
\hline Condiciones atmosfericas & 1 & 1 & 1 & 1 & 1 & 1 & 1 & 1 & 1 & 1 & 1 & 1 & 1 & 1 & 1 & 1 & 1 & 1 & 1 & 1 & 1 & 1 & 1 & 1 \\
\hline Composicion de calidad de aire y el agua & 1 & 1 & 1 & 1 & 1 & 1 & 1 & 1 & 2 & 2 & 2 & 2 & 1 & 2 & 1 & 1 & 1 & 1 & 1 & 1 & 1 & 1 & 1 & 1 \\
\hline Condicion de los recursos ambientales & 1 & 1 & 1 & 1 & 1 & 1 & 1 & 1 & 1 & 1 & 1 & 1 & 1 & 1 & 1 & 1 & 1 & 1 & 1 & 1 & 1 & 1 & 1 & 1 \\
\hline TOTAL & 3 & 3 & 3 & 3 & 3 & 3 & \begin{tabular}{|l|l|}
3 \\
\end{tabular} & 3 & 4 & 4 & 4 & 4 & 3 & 4 & 3 & 3 & 3 & 3 & 3 & 3 & 3 & 3 & 3 & 3 \\
\hline DIMENSIÓN SOCIAL & E1 & E2 & E3 & E4 & E5 & \begin{tabular}{|l|l|} 
E6 \\
\end{tabular} & E7 & E8 & E9 & E10 & \begin{tabular}{|l|l|} 
E11 \\
\end{tabular} & E12 & E13 & \begin{tabular}{|l|l|} 
E14 \\
\end{tabular} & E15 & \begin{tabular}{|l|l|} 
E16 \\
\end{tabular} & E17 & E18 & E19 & E20 & E21 & \begin{tabular}{|l|l|}
$E 22$ \\
\end{tabular} & E23 & E24 \\
\hline Nivel de organización & 1 & 1 & 1 & 1 & 1 & 1 & 1 & 1 & 1 & 1 & 1 & 1 & 1 & 1 & 1 & 2 & 1 & 1 & 1 & 1 & 1 & 1 & 1 & 1 \\
\hline Participación & 1 & 1 & 1 & 1 & 1 & 1 & 1 & 1 & 1 & 3 & 2 & 2 & 2 & 1 & 1 & 2 & 1 & 1 & 1 & 1 & 1 & 1 & 1 & 2 \\
\hline $\begin{array}{l}\text { Grado de relacion ent } \\
\text { comunitarias y las ins }\end{array}$ & 1 & 1 & 1 & 1 & 1 & 1 & 1 & 2 & 2 & 2 & 2 & 2 & 2 & 1 & 1 & 3 & 2 & 1 & 1 & 1 & 1 & 1 & 1 & 1 \\
\hline to comunitario del riesgo & 1 & 1 & 1 & 1 & 1 & 1 & 1 & 1 & 1 & 1 & 1 & 1 & 1 & 1 & 1 & 1 & 1 & 1 & 1 & 1 & 1 & 1 & 1 & 1 \\
\hline TOTAL & 4 & 4 & 4 & 4 & 4 & 4 & 4 & 5 & 5 & 7 & 6 & 6 & 6 & 4 & 4 & 8 & 5 & 4 & 4 & 4 & 4 & 4 & 4 & 5 \\
\hline ENCU & E1 & E2 & E3 & E4 & E5 & E6 & E7 & E8 & E9 & E10 & E11 & E12 & E13 & E14 & E15 & E16 & E17 & E18 & E19 & E20 & E21 & E22 & E23 & E24 \\
\hline \begin{tabular}{|l} 
VALOR TOTAL VULNERABILIDAD \\
\end{tabular} & 25 & 28 & 31 & 33 & 32 & 32 & 33 & 34 & 33 & 34 & 32 & 28 & 26 & 27 & 23 & 32 & 28 & 27 & 30 & 28 & 27 & 27 & 29 & 28 \\
\hline
\end{tabular}

Fuente: Elaboración propia (2016).

\section{Una aproximación a las condiciones de riesgo}

Mediante un análisis cualitativo empleando una matriz de doble entrada (amenazas vs. vulnerabilidad) y el diseño y aplicación de un modelo cartográfico, se determinó las condiciones de riesgo para el área de estudio.

En el corregimiento de Palo de Agua se establecieron dos categorías de riesgo: medio y alto; el centro poblado se encuentra mayoritariamente sobre amenaza media alta y en estas mismas zonas se presentan vulnerabilidad media y baja, por tanto, su riesgo sería medio empleando la relación de la matriz de doble entrada (Figura 5). 
Orlando Tordecilla-Correa, Jorge Villadiego-Lorduy, Rubén Dario Sepulveda-Vargas, Lilliana Piedra-Castro, Ana Michelle Gómez-Constante Vulnerabilidad por erosión fluvial: caso del corregimiento de Palo de Agua, Municipio de Lorica, Departamento de Córdoba, Colombia.

\section{Figura 4: Vulnerabilidad total centro poblado Palo de Agua}

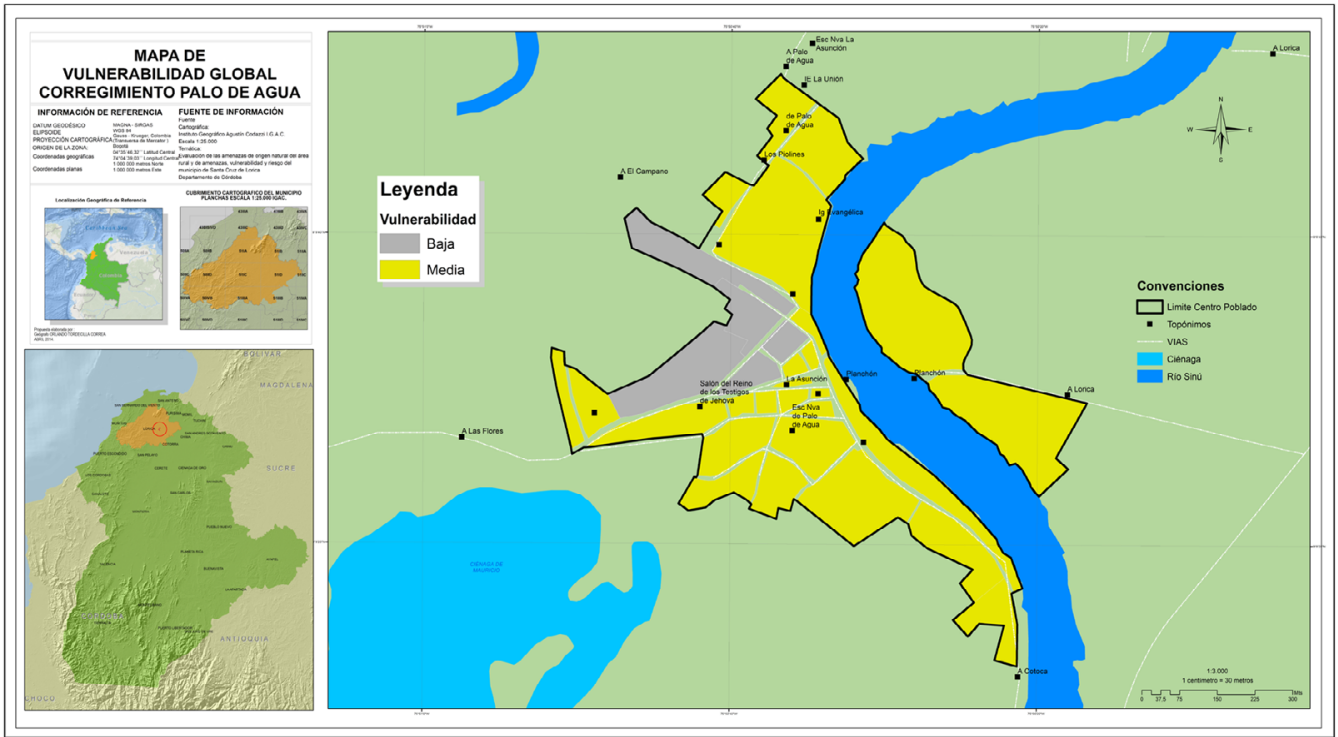

Fuente: Elaboración propia (2016).

Figura 5: Zonificación por riesgo en el corregimiento de Palo de Agua

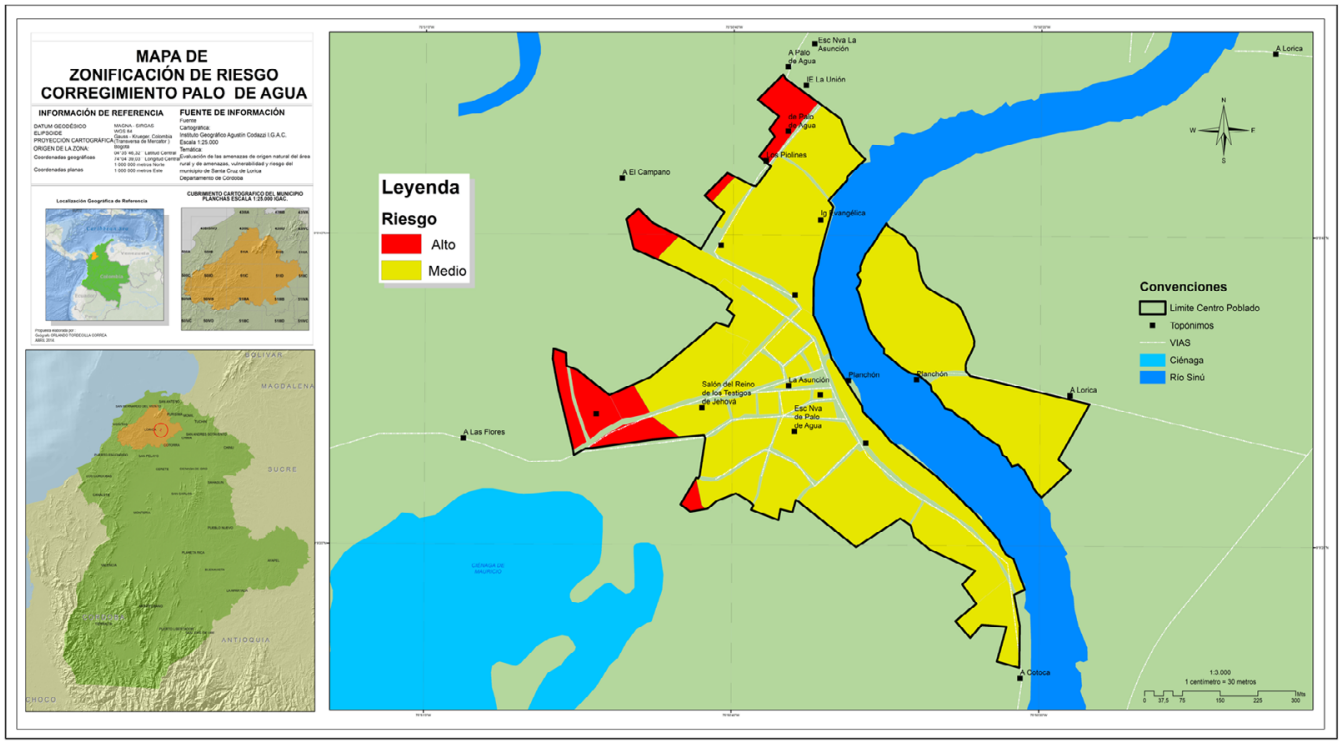

Fuente: Elaboración propia (2016). 
Orlando Tordecilla-Correa, Jorge Villadiego-Lorduy, Rubén Dario Sepulveda-Vargas,

Lilliana Piedra-Castro, Ana Michelle Gómez-Constante

Vulnerability due to fluvial erosion: case of the Palo de Agua township, Municipality of Lorica,

Department of Córdoba, Colombia.

\section{Discusión de resultados}

La erosión fluvial se produce cuando el perfil del río se encuentra en desequilibrio y debido a la energía potencial, gravitatoria y cinética del agua en movimiento, este profundiza el cauce hasta alcanzar su perfil de equilibrio.

Esencialmente, se ha probado en la zona de estudio un aumento en los procesos erosivos fluviales, es importante mencionar que este efecto se intensifica con las inundaciones por desbordamiento, ocasionando la formación de taludes verticales, además de esto, también ha sido evidente un debilitamiento de las orillas del rio, producto de la erosión lateral de las riberas, en algunos casos se ha observado la ruptura de los diques, al igual que la infiltración, el rebose y los represamientos sobre las zonas de afectación.

Cabe resaltar, que el deterioro de las obras de control de inundaciones es notable, incidiendo directamente sobre las inundaciones y en el desarrollo acelerado de los procesos erosivos.

Asociado a esto, las condiciones de infraestructura y socioeconómicas de las poblaciones asentadas en los territorios objeto de estudio son precarias, originando una sinergia de carácter negativo, que favorece la formación de escenarios de vulnerabilidad por erosión fluvial.

Según Lampis (2010, p.66), Foschiatti (2004, p. 13; 2009, p. 263), Salas (2007, p. 35) y Thomas (2013, p. 83) la vulnerabilidad y la pobreza están estrechamente ligadas, la vulnerabilidad se refiere a la composición de atributos de un individuo o individuos, reflejada con relación a la exposición de amenaza que se deriva de la circunstancia social y económica individual o colectiva implicada.

La comunidad de Palo de Agua no es ajena ante esta situación, que se ha convertido en un evento recurrente y sin una solución de fondo y estableciendo una relación directa entre lo señalado por los autores antes mencionados.

La construcción de escenario vulnerables no solo atañe a los corregimientos en estudio, investigaciones de Vidal (2007, p.62) en la zona nororiental de Medellín (Colombia), González (2001) en la ciudad de Buenos Aires (Argentina), Arranz (2008, p. 399) casos de estudio en España, Noriega et al. (2011, p. 99) en su análisis de la vulnerabilidad y el riesgo en la cuenca baja del río Gaira y Cárdenas (2018, p.42) en su análisis de la gestión de riesgo por inundaciones en Colombia, indican que el factor 
antrópico y los procesos deficientes de planificación son elementos comunes frente a la vulnerabilidad y el riesgo.

Por último, los puntos críticos identificados, así como los cálculos estimados de las vulnerabilidades totales para Palo de Agua y la identificación de zonas de riesgo, se convierten en insumos para el ordenamiento y la gestión del riesgo en el territorio.

\section{Conclusiones}

Mediante la investigación realizada en el centro poblado de Palo de Agua, perteneciente al municipio de Santa Cruz de Lorica, se establecen las siguientes conclusiones:

- La erosión fluvial en el corregimiento de Palo de Agua se ha intensificado producto de intervención antrópica en las franjas protectoras. Igualmente, la vulnerabilidad total media es la categoría de mayor predominancia con el $84 \%$ para Palo de Agua.

- Mediante el estudio de campo se logró establecer que las dimensiones que presentan más peso para definir la vulnerabilidad global del corregimiento son la física y la económica con base en los escasos ingresos de la población y las condiciones constructivas de las viviendas. Así mismo, las amenazas por inundación en Palo de Agua no son un fenómeno aislado a los procesos de erosión fluvial presentes en el río Sinú, puesto que este último incide en gran medida en el debilitamiento de las orillas facilitando procesos como la inundación por desborde. De igual forma, el estudio permite orientar la toma de decisiones institucionales para la conservación y el manejo sustentable del recurso suelo, en el marco de los servicios ecosistémicos y disminución de riesgos con medidas de mitigación

\section{Referencias}

Arranz, M. (2008). El riesgo de inundaciones y la vulnerabilidad en áreas urbanas. Análisis de casos en España. Estudios Geográficos, 69(265), pp. 385-416. doi: http://dx.doi.org/10.3989/estgeogr.0417

Cárdenas, K. (2018). Análisis General de la Gestión del Riesgo por Inundación en Colombia. Revista Científica en Ciencias Ambientales y 
Sostenibilidad (CAS). 4(1), pp. 40-45. Disponible en: https://revistas.udea.edu.co/index.php/CAA/article/view/335841

Cáritas del Perú. (2009). Gestión del Riesgo de Desastres para la Planificación del Desarrollo Local. Lima, Perú. Disponible en: https:// www.mesadeconcertacion.org.pe/sites/default/files/guia_de_gestion_de_riesgos.pdf

Corporación Autónoma Regional de los Valles del Sinú y San Jorge - CVS; Universidad del Sinú. (2008). Diagnóstico para la delimitación y caracterización de zonas con amenazas y riesgos. Montería - Córdoba - Colombia.

Corporación Autónoma Regional de los Valles del Sinú y del San Jorge -CVS y Universidad de EAFIT. (2012). Evaluación de las amenazas de origen natural del área rural y de amenazas, vulnerabilidad y riesgo del municipio de Santa Cruz de Lorica - Departamento de Córdoba. Disponible en: https://repositorio.gestiondelriesgo.gov.co/ bitstream/handle/20.500.11762/460/PMGR\%20Santa\%20Cruz $\% 20$ de $\% 20$ Lorica.pdf? sequence $=1 \&$ isAllowed $=y$

Corporación Autónoma Regional de los Valles del Sinú y San Jorge-CVS. (2014). Informe sobre las amenazas de erosión fluvial e inundaciones en la cuenca del río Sinú, departamento de Córdoba. Subdirección de Gestión Ambiental, Grupo de Gestión del Riesgo. Montería - Córdoba - Colombia. Disponible en: https://carbonn.org/uploads/ tx_carbonndata/INFORME\%20GENERAL\%20EROSION\%20 FLUVIAL\%20RIO\%20SINU_CVS_2014_V4_02.pdf

Euroclima. (2014). Guía metodológica cambio climático y gestión del riesgo: análisis de la vulnerabilidad de la infraestructura marinocostera en América Latina. Bruselas, Bélgica: Unión Europea. Disponible en https://euroclimaplus.org/images/ET1_Web.pdf

Defensoría del Pueblo. (2005). Estado Actual de la Cuenca Media y baja del Sinú. Bogotá, Colombia: Defensoría del Pueblo.

Departamento Nacional de Planeación. (2012). Plan Nacional de Adaptación al Cambio Climático. ABC: Adaptación Bases Conceptuales. Marco conceptual y lineamientos. Recuperado de https://www.minambiente.gov.co/images/cambioclimatico/pdf/Plan_nacional_de_ adaptacion/1._Plan_Nacional_de_Adaptaci\%C3\%B3n_al_Cambio_ Clim\%C3\%A1tico.pdf 
DANE. (2017). Boletín técnico. Pobreza Monetaria 2016: Córdoba. Bogotá D.C.: DANE.

Estrategia Internacional para la Reducción de Desastres de las Naciones Unidas (UNISDR). (2009). Terminología sobre Reducción del Riesgo de Desastres. Ginebra, Suiza: Naciones Unidas. Disponible en: https:// www.unisdr.org/files/7817_UNISDRTerminologySpanish.pdf

Foschiatti, A. (2004). Vulnerabilidad global y pobreza- Coonsideraciones conceptuales. Disponible en: https:/hum.unne.edu.ar/revistas/ geoweb/Geo2/archivos/vulglobal.pdf

Foschiatti, A. (2009). Aportes conceptuales y empíricos de la vulnerabilidad global. Disponible en: https://hum.unne.edu.ar/publicaciones/ instGeo/digitales/vulnerabilidades/archivos/cap1.pdf

González, S. (2001). Gestión del riesgo por inundaciones en la ciudad de Buenos Aires Situación actual y alternativa. Revista Realidad Económica, 177. Disponible en: http://www.iade.org.ar/noticias/ gestion-del-riesgo-por-inundaciones-en-la-ciudad-de-buenos-airessituacion-actual-y

Instituto Geográfico Agustín Codazzi - IGAC. (2016). Cartografía base. Colombia. http://www.iade.org.ar/noticias/gestiondel-riesgo-porinundaciones-en-la-ciudad-de-buenos-aires-situacion-actual-y

Lampis, A. (2010). Pobreza y riesgo medioambiental: Un problema de Vulnerabilidad y Desarrollo. Working Paper. Disponible en: https://drive.google.com/file/d/1a0wrkj3_OSLEXZuVP5sI_Njdq8EDCcX1/ view? $\mathrm{usp}=$ sharing

Ministerio de ambiente, Vivienda y Desarrollo territorial (2010). Reglamento Colombiano de Construcción Sismo Resistente - NSR-10. Disponible en: https://www.unisdr.org/files/48578_impactodesastresamericalatinacaribe.pdf

Noriega, O., Gutiérrez, Y., y Rodríguez, J. (2011). Análisis de la vulnerabilidad y el riesgo a inundaciones en la cuenca baja del río Gaira, en el Distrito de Santa Marta. Prospect, 9(2), pp. 93-102. Disponible en: HYPERLINK http://repositorio.uac.edu.co/handle/11619/1275 Ochoa, M. (2011). La Erosión Pluvial y Fluvial y su Incidencia en la Vulnerabilidad de la Terraza Aluvial Sobre la cual está Asentada la Ciudad de Mera, Provincia de Pastaza. Universidad técnica de Ambato, 
facultad de ingeniería civil y mecánica, carrera de ingeniería civil. Ambato - Ecuador.

Oficina de las Naciones Unidas para la Reducción del Riego de Desastres [UNISDR]. (2015). Impacto de los Desastres en América Latina y el Caribe 1990 - 2013. Disponible en: https://www.unisdr.org/files/48578_impactodesastresamericalatinacaribe.pdf.

Programa de Naciones Unidas para el Desarrollo (PNUD). (2014). Informe sobre Desarrollo Humano. Sostener el progreso humano: Reducir Vulnerabilidad y construir resiliencia. New York, EE. UU: PNUD. Disponible en: HYPERLINK "http://hdr.undp.org/sites/default/files/hdr14-summary-es.pdf"

Programa de Naciones Unidas para el Desarrollo Colombia (PNUD) \& Unidad Nacional de Gestión del Riesgo de Desastres (UNGRD). (2012). Guía metodológica para la elaboración de Planes Departamentales para la Gestión del Riesgo. Bogotá, D.C., Colombia.

Salas, J. (2007). Vulnerabilidad, pobreza y desastres 'socionaturales' en Centroamérica y El Caribe. Informes de la Construcción, 59(508), pp. 29-41. Disponible en: HYPERLINK http://digital.csic.es/bitstream/10261/23074/1/662.pdf

Sepúlveda, R. D. (2015). Conflictos socioambientales en la cuenca baja del río Sinú, Colombia. Revista Direitos Emergentes Na Sociedade Global, 4(1), pp. 23-43. https://periodicos.ufsm.br/REDESG/article/view/20377\#.X4RW92hKjIU ADDIN Mendeley Bibliography CSL_BIBLIOGRAPHY

Thomas, J. (2013). Evaluación de la vulnerabilidad social ante amenazas naturales en Manzanillo (Colima). Un aporte de método. Investigaciones Geográficas, 81, pp. 79 - 93. https://www.sciencedirect.com/ science/article/pii/S0188461113727705

Vidal, G. (2007). Evolución de la vulnerabilidad frente a fenómenos asociados con deslizamientos e inundaciones. caso zona nororiental de Medellín, 1960 - 1990. Gestión y Ambiente, 10(2), pp. 53-71. Disponible en: HYPERLINK https://www.redalyc.org/articulo. oa?id=169419816004

Wilches, G. (1993). La vulnerabilidad global. Bogotá, Colombia: Editorial Tercer Mundo. 
Yaima, M. (2018). Diagnóstico de la vulnerabilidad global ante la erosión fluvial e inundaciones de los Consejos Populares Moa Centro y Los Mangos - Joselillo del municipio Moa. Moa, Cuba: Instituto superior minero metalúrgico de Moa de Antonio Núñez Jiménez. 